\title{
(2) OPEN ACCESS \\ Association of Total Sarnat Score with brain injury and neurodevelopmental outcomes after neonatal encephalopathy
}

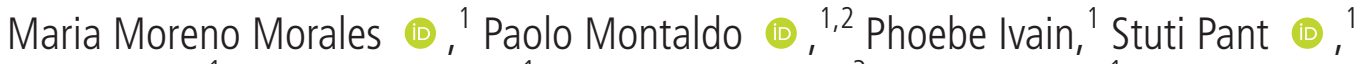 \\ Vijay Kumar, ${ }^{1}$ Vaisakh Krishnan, ${ }^{1}$ Seetha Shankaran, ${ }^{3}$ Sudhin Thayyil ${ }^{1}$
}

\begin{abstract}
- Additional supplemental material is published online only. To view, please visit the journal online (http://dx.doi. org/10.1136/archdischild 2020-321164).
\end{abstract}

${ }^{1}$ Centre for Perinatal

Neuroscience, Brain Sciences Department, Imperial College of Science Technology and Medicine, London, UK

2Department of Neonatal Intensive Care, Università degli Studi della Campania Luigi Vanvitelli, Naples, Italy ${ }^{3}$ Department of Pediatrics, Division of Neonatal and Perinatal Medicine, Wayne State University Childrens Hospital of Michigan, Detroit, Michigan, USA

\section{Correspondence to}

Maria Moreno Morales, Centre for Perinatal Neuroscience, Imperial College of Science Technology and Medicine, London W12 OHS, UK m.moreno-morales@imperial. acuk

Received 12 November 2020 Revised 19 February 2021 Accepted 3 April 2021 Published Online First 5 May 2021

Check for updates

(c) Author(s) (or their employer(s)) 2021. Re-use permitted under CC BY-NC. No commercial re-use. See rights and permissions. Published by BMJ.

To cite: Morales MM, Montaldo $\mathrm{P}$, Ivain $\mathrm{P}$, et al.

Arch Dis Child Fetal Neonatal Ed 2021;106:F669-F672.

\section{ABSTRACT}

We examined the association of Total Sarnat Score (TSS) with brain injury on neonatal magnetic resonance (MR) and adverse neurodevelopmental outcome (NDO) (death or moderate or severe disability) at 2 years of age in 145 infants undergoing therapeutic hypothermia for neonatal encephalopathy. TSS was associated with basal ganglia/thalamic injury on conventional MR $(p=0.03)$ and thalamic N-acetyl aspartate on MR spectroscopy $\left(R^{2}=0.16, p=0.004\right)$ at 2 weeks of age, and Bayley Composite Cognitive $\left(R^{2}=0.18, p=0.01\right)$, Motor $\left(R^{2}=0.15, p=0.02\right)$ and Language $\left(R^{2}=0.11, p=0.01\right)$ Scores at 2 years of age after adjustment for seizures at the time of neurological assessment. The accuracy of TSS (area under the curve $(A U C)=0.71$ ) for predicting adverse NDO was similar to the modified Sarnat staging ( $A \cup C=0.72$ ). TSS of $>12$ within 6 hours of birth indicated high risk of adverse NDO, while TSS of $<4$ indicated intact survival and was reassuring of a good outcome among cooled infants.

\section{BACKGROUND}

Clinical assessment of encephalopathy severity is useful for an early identification of at-risk neonates who may benefit from neuroprotective therapies. Although the original Sarnat staging was based on the evolution of neonatal encephalopathy (NE) over the first few days, it has been subsequently modified and validated as an accurate method for identifying infants with moderate or severe NE who require therapeutic hypothermia $(\mathrm{TH}){ }^{1}$ Over the years, different attempts have been made to improve the accuracy of Sarnat staging to predict neurological outcomes in infants with NE. In the secondary analysis of the National Institute of Child Health and Human Development (NICHD) cooling trial, Ambalavanan et al developed classification trees and a scoring system based on clinical and lab variables. The regression tree model, but not the scoring system, showed a higher accuracy than the early neurological examination alone. ${ }^{2}$ More recently, a new scoring system was developed by adding the numerical values of each component of the Modified Sarnat Score to produce a continuous score. ${ }^{3}$ Whether this continuous scoring system is better than the modified Sarnat staging in infants undergoing $\mathrm{TH}$ is still unknown.

In this secondary analysis of the Magnetic Resonance Biomarkers in Neonatal Encephalopathy

\section{What is already known on this topic?}

- Modified Sarnat staging within 6 hours of birth is useful for predicting later adverse outcomes after neonatal encephalopathy (NE) and for identifying infants with moderate and severe encephalopathy who require therapeutic hypothermia (TH).

- The Total Sarnat Score (TSS) has been recently reported to have a higher prognostic accuracy in non-cooled infants with mild encephalopathy.

\section{What this study adds?}

- TSS is associated with basal ganglia injury on MRI and later adverse neurodevelopmental outcomes in cooled infants with NE.

- The TSS in the first 6 hours of life has prognostic accuracy similar to modified Sarnat staging across all spectrum of infants with encephalopathy undergoing $\mathrm{TH}$.

$\left(\right.$ MARBLE) ${ }^{4}$ study, we studied the accuracy of the Total Sarnat Score (TSS) done within first 6 hours after birth for predicting brain injury and adverse neurodevelopmental outcomes (NDOs) in infants with NE undergoing TH.

\section{METHODS}

The MARBLE study recruited 223 infants born at or after 36 weeks of gestation with NE treated with $\mathrm{TH}$, from eight centres in the UK and the USA. All infants had a structured neurological examination by a certified examiner within 6 hours of birth (on line supplemental table 1). Infants with life-threatening congenital malformations, metabolic disorders, meningitis or encephalitis, and syndromic disorders were excluded.

All infants had MRI and spectroscopy using harmonised sequences at 4-14 days. NDO was assessed at 18-24 months of age by trained and certified examiners by using Bayley Scales of Infant and Toddler Development III examination. ${ }^{5}$ MRI injury was scored by a single central reader using a validated scoring system, masked to the clinical outcomes. ${ }^{6}$

Adverse outcome was defined as death or severe (Bayley III Composite Cognitive and Language 
Scores less than 70, Gross Motor Function Classification System (GMFCS) levels 3-5, hearing impairment requiring hearing aids or blindness) or moderate (composite cognitive and language scores between 70 and 84 and any of the following: hearing impairment without need of amplification, cerebral palsy (GMFCS level 2) and seizure disorder) disability. ${ }^{4}$

For this secondary analysis, TSS was calculated by summating the individual scores from the six categories: level of consciousness, spontaneous activity, tone, posture, primitive reflexes and autonomic system activity. Each category was scored as 0 (normal), 1 (mild), 2 (moderate) or 3 (severe); thus, the score ranged from 0 to 18 .

\section{STATISTICAL ANALYSIS}

We used linear regression to examine the association of TSS with thalamic N-acetyl aspartate (NAA) and Bayley Composite Scores. The area under curve (AUC) of the receiver operating characteristic was used to examine the prognostic accuracy of TSS and to find the best cut-off value to predict neurodevelopment at 2 years. We adjusted the regression model for the presence of seizures at the time of the neurological examination. We used one-way analysis of variance to compare the mean TSSs among the neonates with different basal ganglia/thalami (BGT) and White Matter and Cortex Injury Scores (0, 1, 2 and 3). All analyses were performed with SPSS Statistics V.24, and a p value of $<0.05$ was considered statistically significant.

\section{RESULTS}

TSS within 6 hours of birth and MRI were available in 169 infants, of whom 133 (78\%) had proton MRS including thalamic metabolite peak:area ratios. Twenty-four children were lost to follow-up, and TSS within 6 hours of birth and NDO at 23 months were available for 145 infants. Of these, $27(18 \%)$ infants had mild NE; 103 (71\%) had moderate NE; and 15 (10\%) had severe NE. Adverse NDOs were seen in $21(14.5 \%)$ infants, of whom 1 infant died. The median TSSs of babies with mild, moderate and severe NEs were 5 (IQR 3), 10 (IQR 5) and 16 (IQR 2), respectively (figure 1A). The median age at the time of the MRI scan was 8 (IQR 5) days. In infants with mild, moderate and severe NEs, the median ages at the time of the MRI scan were 7 (IQR 3.75), 8 (IQR 5.0), and 9 (IQR 7.0) days, respectively.

TSS had a significant association with BGT Injury Score groups $(\mathrm{F}=4.78, \mathrm{p}=0.03)$ but not with white matter injury $(\mathrm{F}=0.94$, $\mathrm{p}=0.42)$ and cortex injury $(\mathrm{F}=2.02, \mathrm{p}=0.11)$ (figure $1 \mathrm{~B})$. The TSS was significantly associated with thalamic NAA on magnetic resonance $(\mathrm{MR})$ spectroscopy $\left(\mathrm{R}^{2}=0.09\right.$, equation $=7.67-0.10$, $p=0.005$ ) (figure 1C). After adjustment for seizures at the time of neurological assessment, the association between TSS and thalamic NAA was still statistically significant (adjusted $\left.\mathrm{R}^{2}=0.16, \mathrm{p}=0.04\right)$. The Bayley Composite Cognitive, Motor and Language Scores significantly correlated with the TSS (language $\mathrm{R}^{2}=0.05, \mathrm{p}=009$; motor $\mathrm{R}^{2}=0.08, \mathrm{p}=0.001$; and cognitive $R^{2}=0.07, p=0.001$ ) (figure $1 D$ ). After adjustment for presence of seizures at the time of neurological assessment, the Bayley Composite Cognitive, Motor and Language Scores still significantly correlated with the TSS (language adjusted $\mathrm{R}^{2}=0.11, \mathrm{p}=0.01$; motor adjusted $\mathrm{R}^{2}=0.15, \mathrm{p}=0.02$; and cognitive adjusted $\mathrm{R}^{2}=0.18, \mathrm{p}=0.01$ ).

TSS achieved the best accuracy for prediction of adverse NDO at the cut-off of $>12$ with a $39 \%$ sensitivity $(95 \%$ CI $25 \%$ to $55 \%$ ), $84 \%$ specificity (95\% $75 \%$ to $90 \%$ ) and 0.71 accuracy
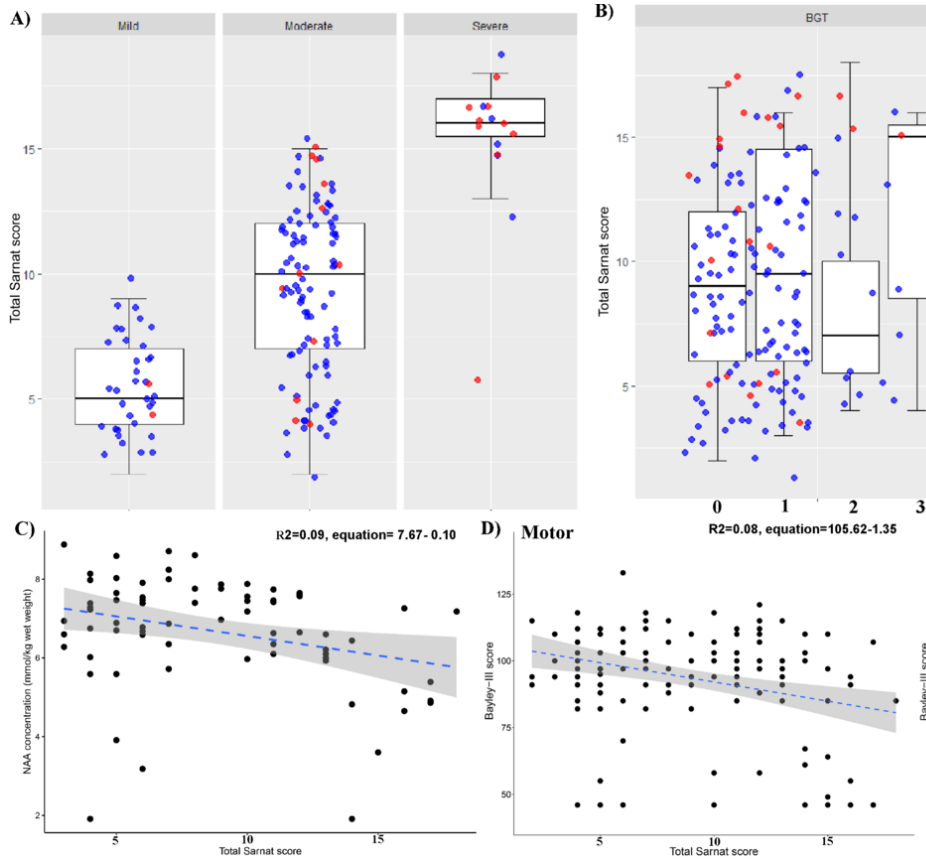
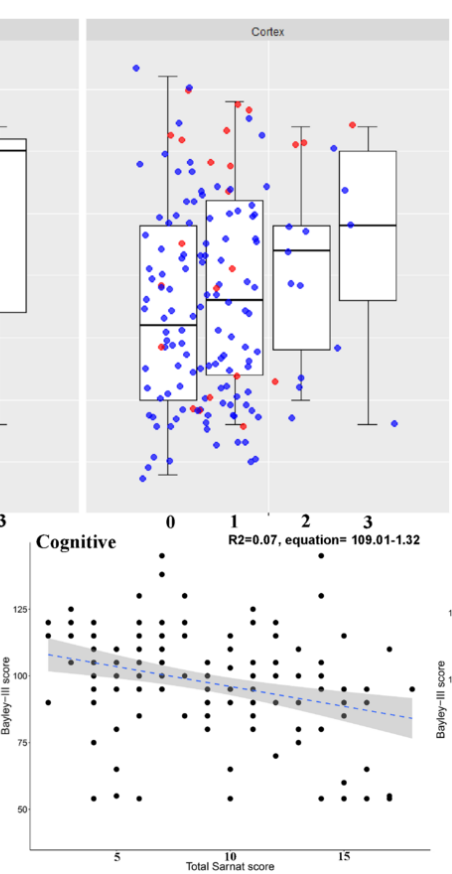
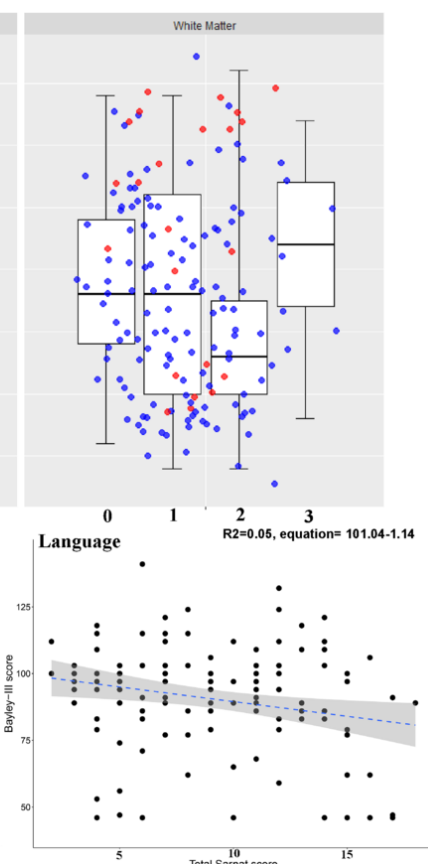

Figure 1 Boxplots exhibiting the spread of TSS across the median and IQR for infants with mild, moderate and severe encephalopathies on admission to the NICU (A) and across BGT, Cortical and White Matter MRI Injury Scores (B). Medians are represented by horizontal lines; the boxes represent the upper and lower quartiles; and the whiskers indicate 1.5×IQR from upper and lower quartiles. Blue dots indicate normal NDO (all Bayley III Composite Scores of $>85$, no disability (hearing or blindness) or persistent seizure disorder at 2 years of age), and red dots are patients with adverse outcomes. Scatterplots exhibiting the association between thalamic NAA (C). Each black dot is a patient and the blue dotted line represents the regression line. Scatterplots demonstrating the association of TSS with cognitive, language and motor scores at all surviving infants at 2 years of age (D). The blue dotted line represents the regression line. BGT, basal ganglia/thalami; NAA, N-acetyl aspartate; NDO, neurodevelopmental outcome; NICU, neonatal intensive care unit; TSS, Total Sarnat Score. 


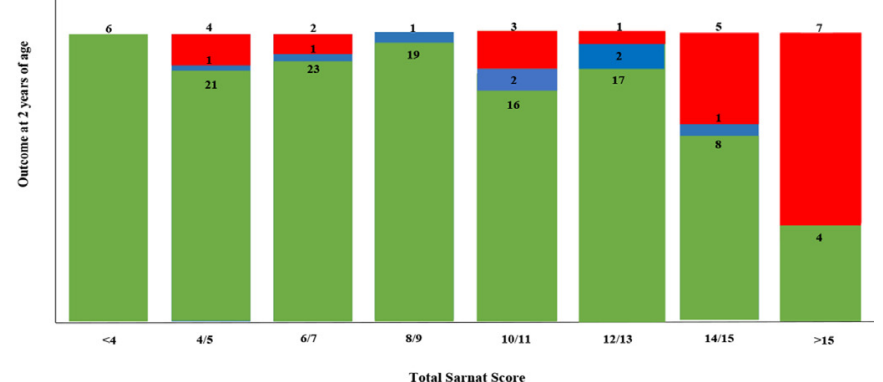

Figure 2 Proportion of normal (represented in green), mild (represented in blue) (cognitive composite score 70-84 or $\geq 85$ and one of the following: GMFCS levels 1-2, seizure disorder or hearing loss) and moderate/severe (represented in red) (Bayley III Composite Score of $<84$ and one or more of the following: GMFCS levels $2-5$, hearing impairment, blindness or persistent seizure disorder) NDOs at 2 years for each TSS. GMFCS, Gross Motor Function Classification System; NDO, neurodevelopmental outcome; TSS, Total Sarnat Score.

(95\% CI 0.57 to 0.93 ). The sensitivity, specificity and AUC of Modified Sarnat Score of $>2$ for predicting NDO was $42 \%$ (95\% CI 25\% to 61\%), 94\% (89\% to $97 \%$ ) and 0.72 (0.65 to 0.78 ), respectively (online supplemental figure 1 ). A TSS of $<4$ indicated intact survival with all Bayley III Composite Scores of $>85$ (figure 2 ).

\section{DISCUSSION}

In this large prospective multicountry cohort of babies with NE treated with TH, TSS calculated within 6 hours after birth significantly correlated with the brain injury on conventional MRI, thalamic MR spectroscopy biomarkers and NDO scores at 2 years of age after adjustment for the presence of seizures at the time of the neurological examination. However, the correlation was weak and $\mathrm{R}^{2}$ values demonstrated extensive spread between outcome measures and the TSS. Overall prognostic accuracy of the score was only modest and similar to the modified Sarnat staging. Nevertheless, extreme values of the TSS are particularly useful in prognostication, with low scores of $<4$ being reassuring and associated with normal outcome and higher scores of $>12$ being associated with more adverse outcomes.

Continuous scores are appealing to clinicians, and hence, Thompson score ${ }^{7}$ is often used in the UK, although it has never been rigorously validated against NDO assessment in large cohorts. The Thompson score has several major limitations, including classification of infants with infrequent seizures as normal or mild, multiple counting of autonomic signs and reliance of examination of anterior fontanelle.

We found that the median TSS of infants with mild and moderate NEs was lower than that of infants with severe NE. However, when neonates with mild and moderate encephalopathy were compared, there was a fair amount of overlap of TSS (figure 1A). This highlights that despite careful clinical assessment using TSS, the differentiation of mild from moderate NE in the first 6 hours still remains challenging.

In the secondary analysis of the Prospective Research on Infants with Mild Encephalopathy (PRIME) study, Chalak et al reported that a TSS of $\geq 5$ had an AUC of 0.83 , a sensitivity of $100 \%$ and a specificity of $67 \%$ in untreated mild NE. ${ }^{8}$ However, our data suggest a much lower prognostic accuracy with a sensitivity of $39 \%$ and a specificity of $82 \%$, possibly reflecting the population differences. Chalak et al recruited only untreated infants with mild NE, while we included all infants (mild, moderate and severe) who had TH. Nevertheless, we found that a TSS of $>12$ within 6 hours after birth indicates a higher risk of adverse NDO, whereas a TSS of $<4$ is indicative of intact survival. The lower prognostic accuracy in infants with TSS between 4 and 12 may reflect the evolution of NE over the first few days, making the predictability of early clinical scoring difficult.

This study has strengths and limitations. First, we assessed only the TSS at the time of admission; therefore, the prognostic accuracy of this tool during TH or afterwards remains unknown. Nevertheless, the neurological examination at the time of admission is the most relevant for clinicians who have to make a decision about the best treatment option within the first 6 hours after birth. Second, our study included only infants who underwent TH. Therefore, it is unknown whether these data would be applicable to non-cooled infants. However, given the widespread use of $\mathrm{TH}$ as standard treatment for moderate or severe encephalopathy and its therapeutic creep in mild NE, these data have direct implications in the clinical setting. Third, our sample size was not sufficiently powered to analyse the accuracy of TSS in the different severity stages of NE. To date, this is the largest multicentre study to analyse the prognostic value of the TSS. Strengths of this study include training and certification of all the examiners, assessment of MRI scans by a single researcher blinded to the admission TSS and that all infants had Bayley score assessment at 2 years of age performed by certified examiners.

In summary, TSS in the first 6 hours of life had similar prognostication as of modified Sarnat staging and correlated with the basal ganglia and thalamic injury. TSS may still be useful for early identification of infants who are more likely to have favourable outcome at 2 years.

Acknowledgements $\mathrm{ST}$ is funded by an advanced fellowship National Institute of Health Research (NIHR); SP is funded by a predoctoral fellowship from the NIHR; and PM is funded by a doctoral fellowship from the Medical Research Council, UK. This research was supported by the NIHR Biomedical Research Centre based at Imperial College London. The views expressed are those of the author and not necessarily those of the NIHR or the Department of Health.

Contributors MMM analysed the data and wrote the original draft of the manuscript. PM and PI supported the data acquisition and interpretation. SP, VKu and $\mathrm{VKr}$ assisted in the interpretation of the data. SS assisted in designing the study and interpreting the data. ST conceived the idea, designed the study, interpreted the data and supervised all aspects of the work.

Funding The study was funded by the National Institute of Health Research, UK.

Competing interests None declared.

Patient consent for publication Not required.

Ethics approval North London Research Ethics Committee (13HH1843).

Provenance and peer review Not commissioned; externally peer reviewed.

Data availability statement Data are available upon reasonable request and once all the different substudies have been published. The data that support the findings of this study are available from the corresponding author, upon reasonable request.

Supplemental material This content has been supplied by the author(s). It has not been vetted by BMJ Publishing Group Limited (BMJ) and may not have been peer-reviewed. Any opinions or recommendations discussed are solely those of the author(s) and are not endorsed by BMJ. BMJ disclaims all liability and responsibility arising from any reliance placed on the content. Where the content includes any translated material, BMJ does not warrant the accuracy and reliability of the translations (including but not limited to local regulations, clinical guidelines, terminology, drug names and drug dosages), and is not responsible for any error and/or omissions arising from translation and adaptation or otherwise.

Open access This is an open access article distributed in accordance with the Creative Commons Attribution Non Commercial (CC BY-NC 4.0) license, which permits others to distribute, remix, adapt, build upon this work non-commercially, 


\section{Short report}

and license their derivative works on different terms, provided the original work is properly cited, appropriate credit is given, any changes made indicated, and the use is non-commercial. See: http://creativecommons.org/licenses/by-nc/4.0/.

\section{ORCID iDs}

Maria Moreno Morales http://orcid.org/0000-0002-1472-6078

Paolo Montaldo http://orcid.org/0000-0002-6034-7976

Stuti Pant http://orcid.org/0000-0002-3912-5965

\section{REFERENCES}

1 Shankaran S, Laptook AR, Ehrenkranz RA, et al. Whole-body hypothermia for neonates with hypoxic-ischemic encephalopathy. N Engl J Med 2005;353:1574-84.

2 Ambalavanan N, Carlo WA, Shankaran S, et al. Predicting outcomes of neonates diagnosed with hypoxemic-ischemic encephalopathy. Pediatrics 2006;118:2084-93.
3 Chalak LF, Adams-Huet B, Sant'Anna G. A total Sarnat score in mild hypoxicischemic encephalopathy can detect infants at higher risk of disability. J Pediatr 2019;214:217-21.

4 Lally PJ, Montaldo P, Oliveira V, et al. Magnetic resonance spectroscopy assessment of brain injury after moderate hypothermia in neonatal encephalopathy: a prospective multicentre cohort study. Lancet Neurol 2019;18:35-45.

5 Lally PJ, Pauliah S, Montaldo P, et al. Magnetic resonance biomarkers in neonatal encephalopathy (marble): a prospective multicountry study. BMJ Open 2015;5:e008912.

6 Rutherford M, Ramenghi LA, Edwards AD, et al. Assessment of brain tissue injury after moderate hypothermia in neonates with hypoxic-ischaemic encephalopathy: a nested substudy of a randomised controlled trial. Lancet Neurol 2010;9:39-45.

7 Thompson CM, Puterman AS, Linley LL, et al. The value of a scoring system for hypoxic ischaemic encephalopathy in predicting neurodevelopmental outcome. Acta Paediatr 1997:86:757-61.

8 A total Sarnat score in mild hypoxic-ischemic encephalopathy can detect infants at higher risk of disability. J Pediatr-Us 2020;214:217. 